\title{
Influence of Moisture and Temperature on Infection of Canada Thistle by Alternaria cirsinoxia
}

\author{
S. Green and K. L. Bailey, Agriculture and Agri-Food Canada, Saskatoon Research Centre, 107 Science Place, \\ Saskatoon, Saskatchewan S7N 0X2, Canada
}

\begin{abstract}
Green, S., and Bailey, K. L. 2000. Influence of moisture and temperature on infection of Canada thistle by Alternaria cirsinoxia. Plant Dis. 84:1126-1132.

Relative humidity (RH), temperature, continuous leaf wetness, and intermittent leaf wetness were evaluated for their influence on conidial germination, appressoria formation, and infection of Canada thistle by Alternaria cirsinoxia. Conidia germinated and formed appressoria at $98 \%$ $\mathrm{RH}$, but required at least $100 \% \mathrm{RH}$, and preferably free water, to penetrate leaves. In free water, conidia germinated equally well at $10,15,20,25$, or $30^{\circ} \mathrm{C}$. Appressoria formation and leaf penetration also occurred at all temperatures evaluated from 10 to $30^{\circ} \mathrm{C}$, with the highest values at 20 to $25^{\circ} \mathrm{C}$ and $20^{\circ} \mathrm{C}$, respectively. Conidia required $8 \mathrm{~h}$ of continuous leaf wetness to establish visible symptoms of infection on Canada thistle. Exposure of conidia of $A$. cirsinoxia to up to five intermittent leaf wetness cycles (30 min wet/30 min dry) reduced germination, appressoria formation, and leaf penetration, but conidia remained infective after all cycles. Exposure to cycles of $4 \mathrm{~h}$ dew/20 h dry was most detrimental to infection, compared with a $72 \mathrm{~h}$ dry period or cycles of $1 \mathrm{~h} \mathrm{dew} / 23 \mathrm{~h}$ wet or $2 \mathrm{~h}$ dew/22 h wet, indicating greater sensitivity of more fully germinated conidia to drying. Such nonspecific temperature requirements and survival during repeated intermittent leaf wetness periods are beneficial characteristics for a bioherbicide. However, high moisture requirements for infection may limit the potential of using A. cirsinoxia to control Canada thistle in the semi-arid prairie provinces of Canada.
\end{abstract}

Additional keywords: Cirsium arvense, infection process

Canada thistle (Cirsium arvense [L.] Scop.) is a perennial weed infesting crop and range lands in the northwestern and northcentral United States and the southern part of all Canadian provinces (10) and was the fifth most abundant crop weed in Saskatchewan, Canada, in 1995 (14). Existing chemical, cultural, and mechanical control measures for this weed usually have limited success, due to its vigor and persistence (10). The application of fungal pathogens as bioherbicides (1) is a potential alternative weed management tool for Canada thistle.

Alternaria cirsinoxia Simmons \& Mortensen is a recently described species isolated from diseased Canada thistle in Saskatchewan, Canada (13). A. cirsinoxia can cause severe foliar necrosis of Canada thistle and has been selected for evaluation as a candidate bioherbicide for this weed by Agriculture and Agri-Food Canada in Saskatoon. To develop A. cirsinoxia as a bioherbicide, it is necessary to understand

Corresponding author: S. Green

E-mail: greens@em.agr.ca

Accepted for publication 5 July 2000.

Publication no. D-2000-0819-01R

This article is in the public domain and not copyrightable. It may be freely reprinted with customary crediting of the source. The American Phytopathological Society, 2000. the ecological requirements of this fungus for infection and survival on Canada thistle. Available water and, to a lesser extent, temperature, are key environmental factors regulating germination and growth of fungi (2). In general, Alternaria spp. are highly resistant to adverse weather, can develop under a wide range of temperatures, and can utilize locally available sources of moisture, hence their ubiquity (12). However, these characteristics have not been defined specifically for A. cirsinoxia.

Saskatchewan is the driest province in Canada, with infrequent rainfall and low relative humidity. Although short dew periods occur on cooler nights, leaf surface moisture tends to be lost rapidly, as irradiance and evapotranspiration levels are high (5). This results in prolonged dry periods interspersed with short cycles of wetting and drying. Since A. cirsinoxia is endemic on Canada thistle in Saskatchewan, it should be well adapted to survive in this climate, but the ability of this fungus to cause high levels of disease sufficient for controlling Canada thistle under these conditions is unknown. Ecological characteristics likely to be important in the success of A. cirsinoxia as a bioherbicide include the ability to infect Canada thistle at relative humidities below saturation point and/or during short dew periods, nonspecific temperature requirements, and survival during repeated cycles of desiccation and rehydration.
This study was conducted to determine the effects of relative humidity, temperature, and continuous and intermittent leaf wetness periods on germination, appressoria formation, and infection of Canada thistle by $A$. cirsinoxia.

\section{MATERIALS AND METHODS}

Plant preparation. Canada thistle plants were grown from rootstocks taken from a naturally occurring field infestation at the Agriculture and Agri-Food Canada Research Farm, near Saskatoon, Saskatchewan, during the previous growing season, and stored at $4{ }^{\circ} \mathrm{C}$ until use. Rootstocks were rinsed in tap water, cut into 8to $10-\mathrm{cm}$ lengths, and planted in a soilless mix (one part sand to four parts of a mixture consisting of approximately $30 \%$ sphagnum peat moss, $70 \%$ vermiculite, $1 \%$ calcium carbonate, and 1\% 16-8-12 slowrelease fertilizer) in $10 \mathrm{~cm}$-diameter plastic pots. Plants were grown in a greenhouse at $20 \pm 3{ }^{\circ} \mathrm{C}$ with supplementary lighting provided with 430 watt high-pressure sodium lamps $\left(230 \mu \mathrm{E} \cdot \mathrm{m}^{-2} \cdot \mathrm{s}^{-1}\right)$ (Philips Son-Agro) for about 6 weeks until the 8 to 10 leaf growth stage. For the experiments evaluating relative humidity and temperature, excised leaves were used, with the first fully expanded, nonsenescent leaf selected from the base of each plant. To ensure that any heterogeneity among Canada thistle plants was spread randomly among treatments, each excised leaf was taken from a different plant and leaves randomly assigned to each treatment. Whole plants were used in all other experiments and randomly assigned to treatments.

Inoculum preparation and plant inoculation. A single isolate of A. cirsinoxia, 93-109 B1 (obtained from the Saskatoon Research Centre culture collection) was used in this study. The isolate was originally collected from Canada thistle in Watrous, Saskatchewan, in 1993. Stock cultures were maintained as conidia frozen in $5 \%$ skim milk and $20 \%$ glycerol at $-73^{\circ} \mathrm{C}$. Conidia were produced using the methodology described by Walker (15) and were added to distilled water to give a suspension of $10^{5}$ conidia/ml, determined using a hemacytometer. The conidial suspension was stirred to prevent clumping of conidia and sprayed immediately after preparation onto excised leaves or whole plants using a cabinet sprayer (model RP1, Ratiopax, Incom International Inc.) set at $250 \mathrm{kPa}$ constant air pressure. The cabinet 
sprayer speed was calibrated to deliver an amount of inoculum equivalent to a field rate of 400 liters/ha by spacing the excised leaves or whole plants evenly within the spray chamber and subjecting them to two consecutive spray passes. This meant that all treatments received a standardized, even coverage of inoculum without leaf surface run-off.

Assessment of conidial germination, appressoria formation, leaf penetration, and disease development on whole plants. To assess conidial germination, appressoria formation, and leaf penetration, leaf disks were cut from the mid-leaf area of the oldest, fully expanded, nonsenescent leaf at the base of each plant inoculated using a 10-mm-diameter cork borer. For each experiment, all treatments were sampled in quick succession and the leaf disks stained immediately to halt infection. To evaluate conidial germination, leaf disks were stained with a $10-\mu$ droplet of lactophenol cotton blue (Fisher Scientific) and examined microscopically. Approximately 100 conidia per disk were counted within fields of view selected from left to right across each disk. Conidia were considered to have germinated when the germ tube was at least half the width of the conidium. The number of germinated conidia was expressed as a percentage of the total number of conidia counted. To evaluate appressoria formation and leaf penetration, leaf disks were cleared and stained for $24 \mathrm{~h}$ in a solution of Chlorazole Black E (Sigma Chemical Company) and destained for $24 \mathrm{~h}$ in a saturated solution of chloral hydrate, as described by Keane et al. (9). Destained disks were mounted in $50 \%$ glycerine and examined microscopically. The total number of appressoria formed, and the total number of leaf penetration sites, were counted and added for approximately 100 germinated conidia per disk in fields of view selected as described above. Successful leaf penetration was determined by the presence of an infection hypha in the epidermal cell below an appressorium, and leaf penetration values were based on the sum of all appressoria forming an infection hypha for every germinated conidium counted. Appressoria formation and leaf penetration values were divided by the number of germinated conidia counted and expressed as a number per germinated conidium. This species is observed to form multiple germ tubes from a single conidium, and therefore more than one appressorium per conidium may develop. Disease development on whole plants was assessed 14 days after inoculation using the 0 to 11 Horsfall-Barratt rating scale (8) whereby $0=0 \%$ diseased and $11=100 \%$ diseased.

Effect of relative humidity. Inoculated, excised leaves were placed on a wire rack suspended above a reservoir of distilled water or saturated salt solutions of $\mathrm{K}_{2} \mathrm{SO}_{4}$, $\mathrm{MgSO}_{4} \cdot 7 \mathrm{H}_{2} \mathrm{O}, \mathrm{KCl}$, or $\mathrm{NaCl}$ in sealed 0.9 liter plastic humidity chambers to provide $100,98,90,85$, or $75 \% \mathrm{RH}$ environments, respectively (16). A free water treatment consisted of placing inoculated leaves inside a chamber containing distilled water, misting the leaf surfaces with distilled water, and sealing the chamber with parafilm for $24 \mathrm{~h}$. This provided a continuous film of free water on the leaf surface. There was a single chamber for each humidity treatment and four leaves were placed in each chamber as soon as the inoculum had dried on the leaf surface. To assess the effect of RH on ungerminated conidia, inoculated leaves were placed in the chambers and transferred to an incubator set at $20^{\circ} \mathrm{C}$ with no light. After $24 \mathrm{~h}$, conidial germination, appressoria formation, and leaf penetration were assessed as described above. To assess the effect of RH on germinated conidia, inoculated leaves were placed in a dew chamber (model E54U-DL, Percival, Boone, IA) for $4 \mathrm{~h}$ with continuous leaf wetness at $20 \pm 2{ }^{\circ} \mathrm{C}$ and continuous darkness to initiate germination of the conidia. Inoculated leaves were then transferred to the RH chambers and placed in an incubator set at $20^{\circ} \mathrm{C}$ with no light. After $24 \mathrm{~h}$, appressoria formation and leaf penetration were assessed as described above. Each experiment was conducted three times.

Effect of temperature. Inoculated, excised leaves were placed in sealed moisture chambers, with four leaves per chamber. A moisture chamber consisted of a glass petri dish containing water-saturated filter paper. The leaf surfaces were misted with distilled water and the petri dishes sealed with parafilm for $24 \mathrm{~h}$. This provided a continuous film of free water for $24 \mathrm{~h}$. Moisture chambers were placed in incubators set at $10,15,20,25$, and $30^{\circ} \mathrm{C}$ for $24 \mathrm{~h}$ with no light, after which conidial germination, appressoria formation, and leaf penetration were assessed as described above. The experiment was conducted three times.

Effect of continuous leaf wetness period. Inoculated whole plants were placed in a dew chamber at $20 \pm 2{ }^{\circ} \mathrm{C}$ with continuous darkness, for $0,2,4,8,24,32$, or $48 \mathrm{~h}$ before being transferred to a growth chamber (model GR48, Conviron, Winnipeg, Canada). The growth chamber was maintained at $24 / 15^{\circ} \mathrm{C}$ day/night temperatures with a $16 \mathrm{~h}$ photoperiod $\left(500 \mu \mathrm{E} \cdot \mathrm{m}^{-2} \cdot \mathrm{s}^{-1}\right)$ and approximately 50 to $70 \%$ relative humidity (RH), and water was added directly to the potting mix with a hose to avoid wetting the leaves. Conidial germination, appressoria formation, and leaf penetration were assessed $48 \mathrm{~h}$ after inoculation. Plants were maintained in the growth chamber for 14 days, after which disease development was assessed. The experiment was conducted twice, with four replicate plants per treatment.

Effect of intermittent leaf wetness periods. Two experiments were conducted in which inoculated plants were subjected to different intermittent leaf wetness periods. An intermittent leaf wetness period consisted of a dew period followed by a dry period. The experiments were designed to test whether conidia can survive and infect Canada thistle after exposure to either short intermittent leaf wetness periods in rapid succession, or long dry periods following dew periods of increasing length. For each dew period, plants were misted with distilled water and placed in a dew chamber at $20 \pm 2^{\circ} \mathrm{C}$ with continuous darkness. For each dry period, plants were dried with cool blown air circulated by a fan inside the growth chamber. In the first experiment, inoculated plants were given one, two, three, four, five, or no intermittent leaf wetness cycles of $30 \mathrm{~min}$ dry/30 min dew before being placed in the dew chamber for $24 \mathrm{~h}$. In the second experiment, inoculated plants were given one of the following treatments: (i) a 24-h continuous leaf wetness period (referred to as no intermittent leaf wetness); (ii) a 72-h dry period followed by $24 \mathrm{~h}$ continuous leaf wetness; (iii) three cycles of $1 \mathrm{~h}$ dew/23 h dry, followed by $24 \mathrm{~h}$ continuous leaf wetness; (iv) three cycles of $2 \mathrm{~h}$ dew/22 h dry, followed by $24 \mathrm{~h}$ continuous leaf wetness; and (v) three cycles of $4 \mathrm{~h}$ dew/20 h dry, followed by $24 \mathrm{~h}$ continuous leaf wetness. Conidial germination, appressoria formation, and leaf penetration were assessed after the 24-h continuous leaf wetness period for each treatment. Plants were then transferred to the growth chamber for 14 days, after which disease development was assessed. The growth chamber conditions were set as described for the continuous leaf wetness experiment. Each experiment was conducted twice with four replicate plants per treatment.

Experimental design and data analysis. A completely randomized design was used in the experiments on RH and temperature, with individual leaves in each treatment as replicate subsamples and the three trials serving as replicates in time. A randomized complete-block design was used in the experiments on continuous leaf wetness and intermittent leaf wetness, in which the plants were blocked by replicate according to spatial arrangement in the dew chamber and growth chamber. Since the variances were not independent of the means in all experiments, data were arcsin transformed before analysis. Univariate analyses were used to check the normality of distribution of transformed data, and this showed that data were normally distributed in all experiments. The experiments evaluating $\mathrm{RH}$ and intermittent leaf wetness were analysed using analysis of variance (ANOVA) (PROC GLM procedure, SAS Institute version 6.12, Cary, NC) to determine the significance of the main factors and their interactions. When the ANOVA showed significant differences among treatments at $P<0.05$, separation of means 


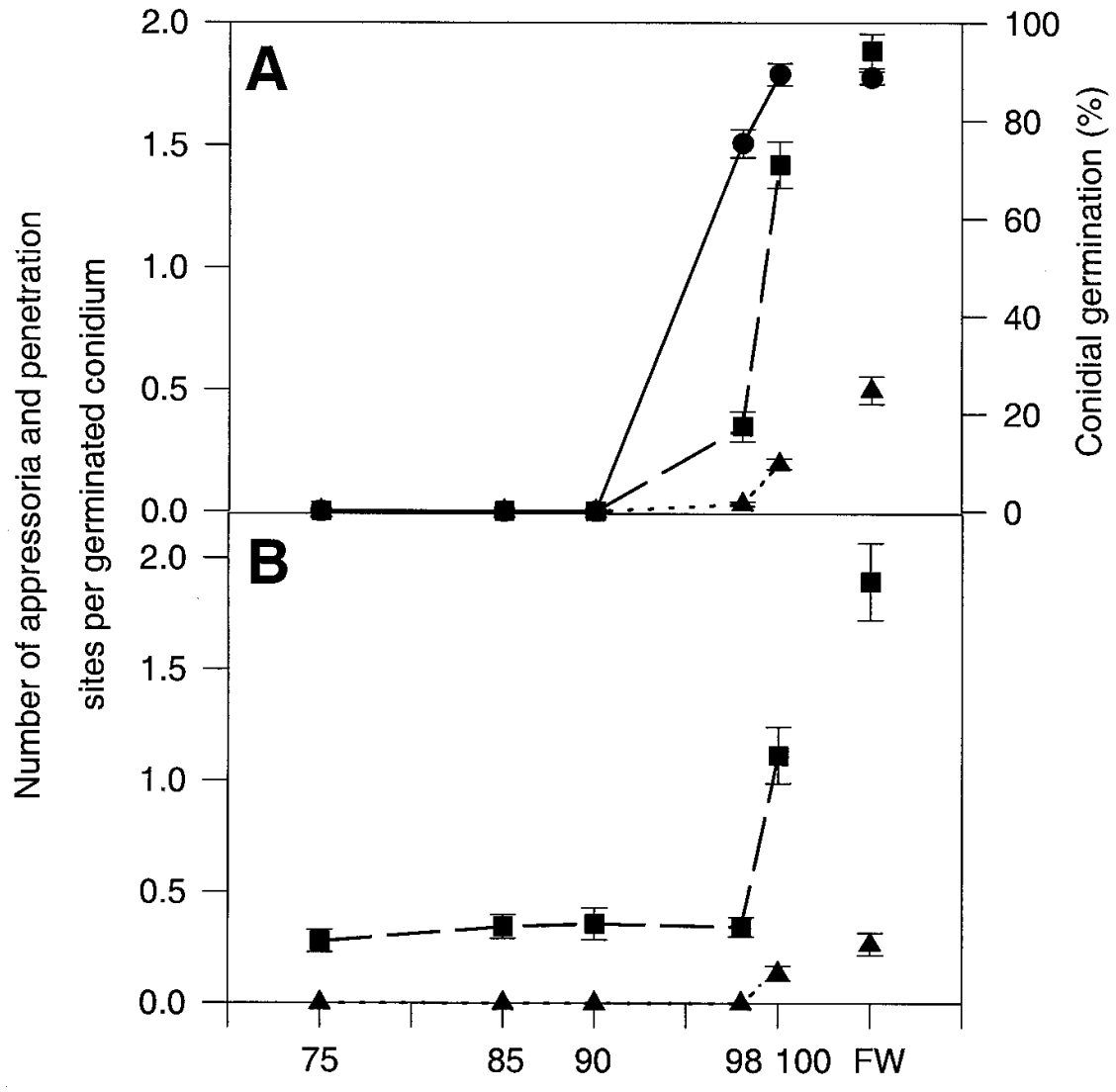

Relative humidity (\%) or free water on the leaf (FW)

Fig. 1. Effects of different levels of relative humidity $(\mathrm{RH})$ and the presence of free water $(\mathrm{FW})$ on the infection process of Alternaria cirsinoxia on Canada thistle $24 \mathrm{~h}$ after inoculation. (A) Germination ( $)$, appressoria formation $(\boldsymbol{\square})$, and leaf penetration $(\boldsymbol{\Delta})$ by conidia ungerminated prior to exposure to the RH and FW treatments; (B) appressoria formation (ם) and leaf penetration ( $\mathbf{\Delta})$ by conidia germinated prior to exposure to the RH and FW treatments. Data presented are untransformed means and bars indicate standard error.
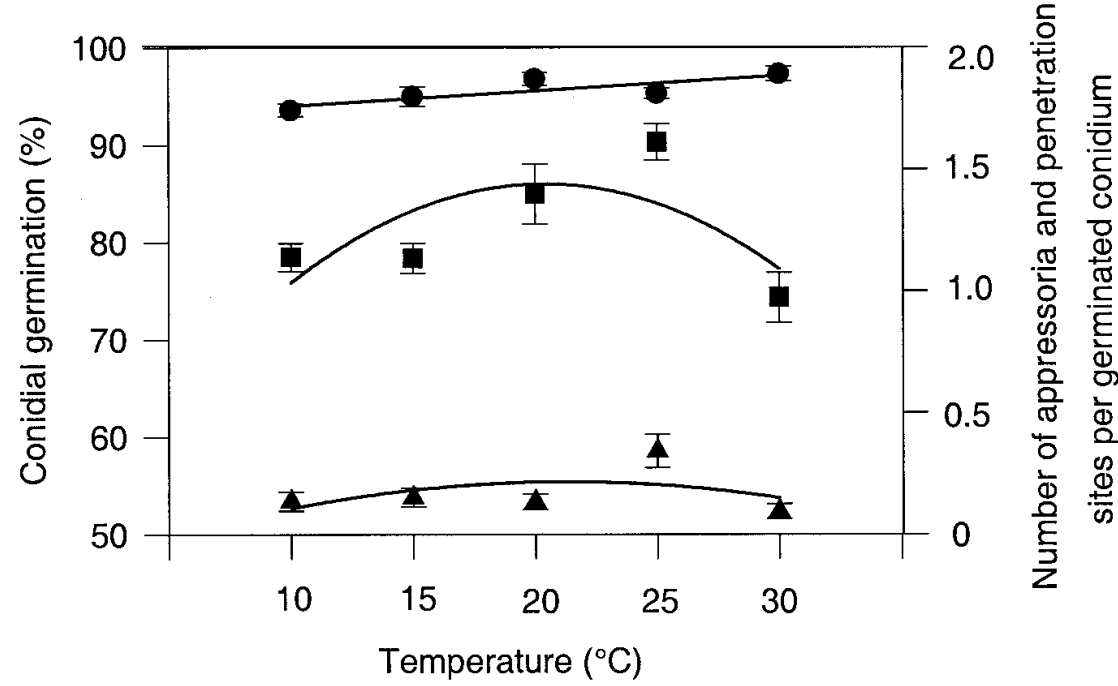

Fig. 2. Regression models for the effect of temperature on germination (-) (no significant function) of conidia of Alternaria cirsinoxia, appressoria formation ( $(Y)\left(Y=0.26-0.03 x+0.002 x^{2}, r_{\text {adj }}^{2}=\right.$ $0.33, P<0.001)$, and penetration of Canada thistle leaves $(\mathbf{\Lambda})\left(Y=0.18-0.03 x+0.002 x^{2}, r_{\text {adj }}^{2}=\right.$ $0.12, P<0.05) 24 \mathrm{~h}$ after inoculation. Data presented are untransformed means and bars indicate standard error. was conducted using the least significant difference (LSD) at $P<0.05$. The experiments evaluating temperature and continuous leaf wetness were evaluated using ANOVA to determine the significance of the main factors and their interactions, and regression analysis of the individual data points was used to fit the linear models (PROC REG procedure, SAS Institute version 6.12, Cary, NC). The regression equations presented in the figure captions are for the individual data points; however, for presentation purposes the graphs shown illustrate the means and regression trends. Standard errors of untransformed means were calculated.

\section{RESULTS}

Effect of relative humidity. Humidity treatment had a significant effect on all variables for both ungerminated and pregerminated conidia of A. cirsinoxia (ANOVA, $P<0.05$ ). Conidia did not germinate at or below $90 \% \mathrm{RH}$, whereas $75 \%$ of the conidia germinated at $98 \% \mathrm{RH}$, and $89 \%$ of the conidia germinated at $100 \%$ RH (Fig. 1A). Appressoria formation occurred at $98 \% \mathrm{RH}$ with 0.4 appressoria per germinated conidium. Appressoria formation increased to 1.4 appressoria per germinated conidium at $100 \% \mathrm{RH}$ and was highest in free water, with 1.9 appressoria per germinated conidium (Fig. 1A). Incidence of leaf penetration was rare at $98 \%$ $\mathrm{RH}$, but increased to 0.2 penetration sites per germinated conidium at $100 \% \mathrm{RH}$, and was highest in free water with 0.5 penetration sites per germinated conidium (Fig. 1A). Germinated conidia formed approximately 0.3 appressoria per conidium after exposure to a 4-h dew period followed by $75,85,90$, or $98 \%$ RH (Fig. 1B). Appressoria formation increased to 1.1 appressoria per germinated conidium at $100 \% \mathrm{RH}$ and was highest in free water, with 1.9 appressoria per germinated conidium (Fig. 1B). Pre-germinated conidia required $100 \% \mathrm{RH}$ to penetrate leaves, with 0.1 penetration sites per germinated conidium. The highest incidence of leaf penetration occurred in free water, with 0.3 penetration sites per germinated conidium (Fig. 1B).

Effect of temperature. The ANOVA showed that temperature had a significant effect on germination of conidia of $A$. cirsinoxia, appressoria formation, and leaf penetration $(P<0.01)$. The effects of temperature on all three variables are presented as regressions of means against temperature (Fig. 2). Germination, appressoria formation and leaf penetration occurred at all temperatures tested (Fig. 2). There was no significant function that fitted germination, with 94 to $97 \%$ germination at all temperatures tested (Fig. 2). Appressoria formation varied from approximately 1.0 appressoria per germinated conidium at 10,15 , and $30^{\circ} \mathrm{C}$, to 1.4 and 1.6 appressoria per germinated conidium at 20 and $25^{\circ} \mathrm{C}$, respectively (Fig. 2). Values 
for leaf penetration ranged from approximately 0.1 penetration sites per germinated conidium at $10,15,20$, and $30^{\circ} \mathrm{C}$ to 0.3 penetration sites per germinated conidum at $25^{\circ} \mathrm{C}$ (Fig. 2). The quadratic function provided a good fit for both appressoria formation $(P=0.006)$ and leaf penetration $(P=0.017)\left(r^{2}=0.33\right.$ and 0.12 , respectively), with both curves showing a slight increase related to the highest values of appressoria formation and leaf penetration at 20 to $25^{\circ} \mathrm{C}$ and $20^{\circ} \mathrm{C}$, respectively (Fig. 2).

Effect of continuous leaf wetness period. The ANOVA showed a significant interaction $(P<0.01)$ between repeated experiments (trials) and continuous leaf wetness treatment, so each trial was analyzed separately. In both trials, continuous leaf wetness period had a significant effect on germination of conidia of A. cirsinoxia, appressoria formation, leaf penetration, and disease rating on whole plants of Canada thistle at 14 days (ANOVA, $P<$ $0.001)$. The effects of continuous leaf wetness period on all four variables are presented as regressions of means against continuous leaf wetness period (Fig. 3A and $\mathrm{B}$ ). Germination of conidia was best described by quadratic functions in both trial one $(P=0.01)$ and trial two $(P=$ $0.0002)\left(r^{2}=0.46\right.$ and 0.73 , respectively) (Fig. 3A and B). Germination began during $2 \mathrm{~h}$ continuous leaf wetness, and reached $90 \%$ after $4 \mathrm{~h}$ continuous leaf wetness in trial one and $8 \mathrm{~h}$ continuous leaf wetness in trial two (Fig. 3A and B). Cubic functions provided a good fit for appressoria formation in both trial one $(P=0.0001)$ and trial two $(P=0.002)\left(r^{2}=0.84\right.$ and 0.70 , respectively) (Fig. 3B). Appressoria formation was first observed after $2 \mathrm{~h}$ continuous leaf wetness in both trials, and increased up to $8 \mathrm{~h}$ continuous leaf wetness, after which 2.0 and 1.3 appressoria per germinated conidium were formed in trials one and two, respectively (Fig. 3A and B). In both trials, continuous leaf wetness periods of 24 and $32 \mathrm{~h}$ did not increase appressoria formation further. However, appressoria formation increased again to 2.7 and 1.9 appressoria per germinated conidium after $48 \mathrm{~h}$ continuous leaf wetness in trials one and two, respectively (Fig. 3A and B). Leaf penetration increased linearly with increasing continual leaf wetness period in trial one $\left(P=0.0006, r^{2}=0.88\right)$, but there was no significant function that fitted leaf penetration in trial two (Fig. 3A and B). In both trials, leaf penetration was first observed after $4 \mathrm{~h}$ continuous leaf wetness, with 0.7 penetration sites per germinated conidium after $48 \mathrm{~h}$ continuous leaf wetness (Fig. 3A and B). There was no significant function that fitted disease rating on whole plants at 14 days in either trial (Fig. $3 C)$. Eight hours of continuous leaf wetness was required for $A$. cirsinoxia to establish visible infection on Canada thistle in both trials (Fig. 3C). In trial one, plants given $8,24,32$, or $48 \mathrm{~h}$ continuous leaf wetness scored 4 to 5 (12 to $50 \%$ diseased) on the Horsfall-Barratt disease rating scale at 14 days after inoculation (Fig. 3C). In trial two, plants given $8 \mathrm{~h}$ continuous leaf wetness scored 3 (6-12\% diseased) on the Horsfall-Barratt disease rating scale, whereas plants given 24,32 , and 48 h continuous leaf wetness had mean disease severity ratings of 5 to 6 (25 to $75 \%$ diseased) (Fig. 3C).
Effect of intermittent leaf wetness periods. In both experiments, the ANOVA showed no significant interaction between trials and intermittent leaf wetness treatment, so data from each trial were pooled for analysis. Intermittent leaf wetness treatment had a significant effect on germination of conidia of $A$. cirsinoxia, appressoria formation, leaf penetration, and disease rating on whole plants of Canada thistle at 14 days, in both experiments

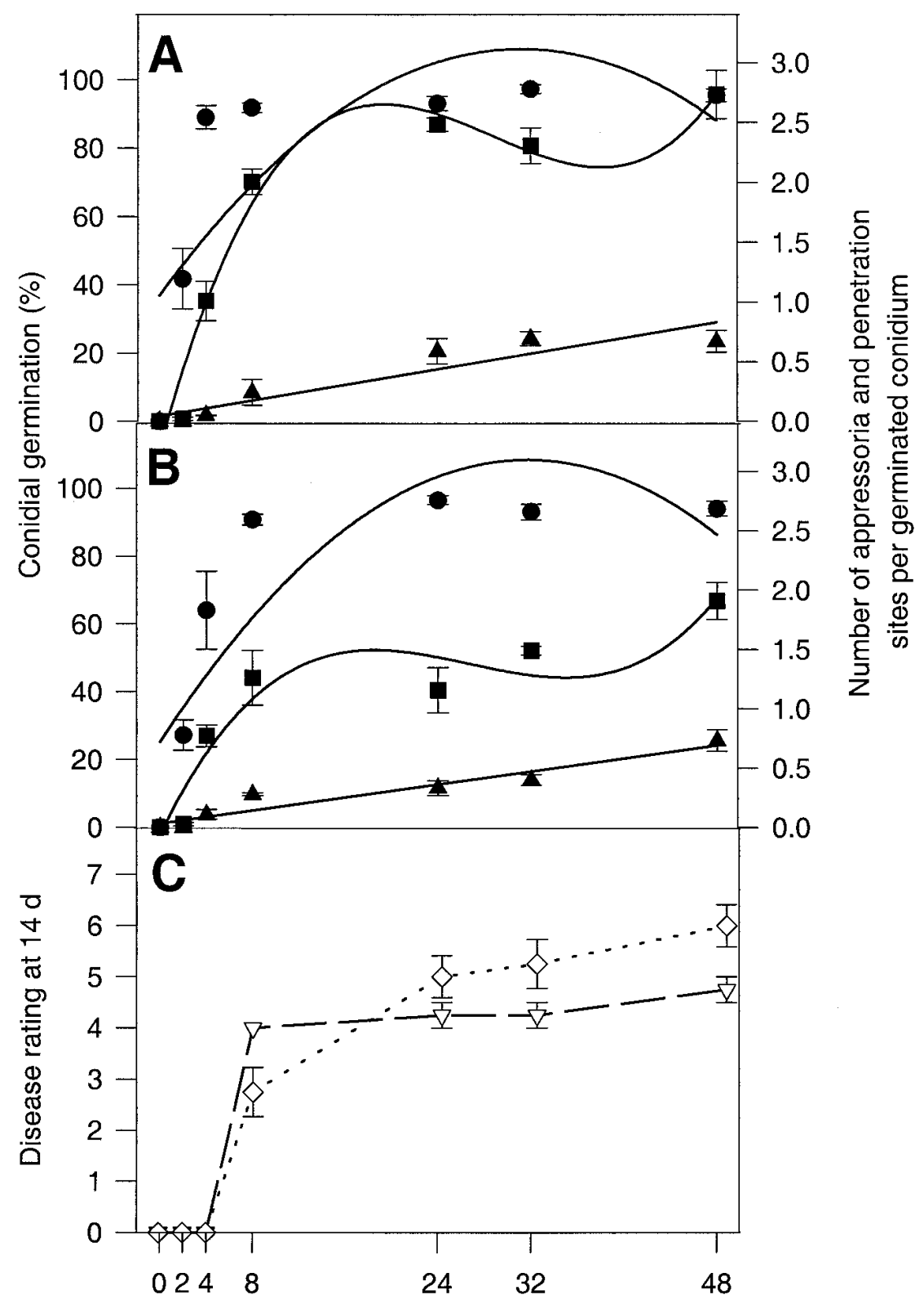

Continuous leaf wetness (hours)

Fig. 3. Regression models for the effect of continuous leaf wetness period on the infection process of Alternaria cirsinoxia on Canada thistle. (A) Germination of conidia $(-)(Y=0.06+0.005 x-$ $\left.0.0002 x^{2}, r_{\text {adj }}^{2}=0.46, P<0.01\right)$, appressoria formation (⿴) $\left(Y=-0.01-0.03 x-0.001 x^{2}+0.00001 x^{3}\right.$, $\left.r^{2}{ }_{\text {adj }}=0.84, P<0.001\right)$, and leaf penetration $(\mathbf{\Delta})\left(Y=0.02+0.009 x, r^{2}=0.88, P<0.001\right), 48 \mathrm{~h}$ after inoculation in trial 1; (B) germination of conidia $(\bullet)\left(\mathrm{Y}=0.04+0.008 x-0.0003 x^{2}, r^{2}\right.$ adj $=0.73, P<$ $0.001)$, appressoria formation $\square\left(Y=-0.009+0.02 x-0.0007 x^{2}+0.000008 x^{3}, r^{2}\right.$ adj $=0.70, P<$ 0.001 ), and leaf penetration $\boldsymbol{\Delta}$ (no significant function), $48 \mathrm{~h}$ after inoculation in trial 2; (C) disease ratings on whole plants 14 days after inoculation in trial $1(\nabla)$ (no significant function) and trial 2 $(\diamond)$ (no significant function). Data presented are untransformed means and bars indicate standard error. 
(ANOVA, $P<0.05$ ). In general, increasing the number and duration of intermittent leaf wetness cycles decreased the viability of $A$. cirsinoxia on Canada thistle, although the pathogen was still able to establish infection on plants after all intermittent leaf wetness cycles (Figs. 4 and 5). In the first experiment, where inoculated plants were given 30 min intermittent leaf wetness cycles, germination was $88 \%$ or higher after $0,1,2,3$, or 4 intermittent leaf wetness cycles, decreasing to $70 \%$ only after 5 intermittent leaf wetness cycles (Fig. 4A). Appressoria formation and leaf penetration were highest (1.6 to 1.9 appressoria and 0.5 penetration sites per germinated conidium) after 0 or 1 intermittent leaf wetness cycle, and lowest (1.0 appressoria and 0.03 penetration sites per germinated conidium) after 4 or 5 intermittent leaf wetness cycles (Fig. 4A). Plants given 5 intermittent leaf wetness cycles also had the lowest mean disease severity rating of 3 (6 to $12 \%$ diseased), 14 days after inoculation, whereas all other intermittent leaf wetness treatments resulted in disease ratings of 4 to 5 (12 to $50 \%$ diseased) (Fig. $4 \mathrm{~B})$. In the second experiment, germination was reduced from values of 90 to $97 \%$ after the $1 \mathrm{~h}, 2 \mathrm{~h}$, or no intermittent leaf wetness cycles to $61 \%$ by the $4 \mathrm{~h}$ intermittent leaf wetness cycle (Fig. 5A). Appressoria formation and leaf penetration were highest (1.8 appressoria and 0.7 penetration sites per germinated conidium) when not exposed to any intermittent leaf wetness period, and lowest ( 0.9 appressoria and 0.01 penetration sites per germinated conidium) after the 2- and 4-h intermittent leaf wetness cycles, respectively (Fig. 5A). The 72-h dry period reduced appressoria formation and leaf penetration compared with no intermittent leaf wetness (Fig. 5A). Disease severity on plants decreased with increasing duration of intermittent leaf wetness, with the highest mean disease severity scores of 5 to 6 ( 25 to $75 \%$ diseased) after no intermittent leaf wetness or a 72-h dry period, and the lowest mean disease severity score of 2 (3 to $6 \%$ diseased) after the 4-h intermittent leaf wetness cycles (Fig. 5B).

\section{DISCUSSION}

The ecological adaptation of $A$. cirsinoxia to the climate of the Canadian prairies is essential to its success as a bioherbicide for Canada thistle in this region. This study shows that A. cirsinoxia requires moisture levels at, or close to, satu-

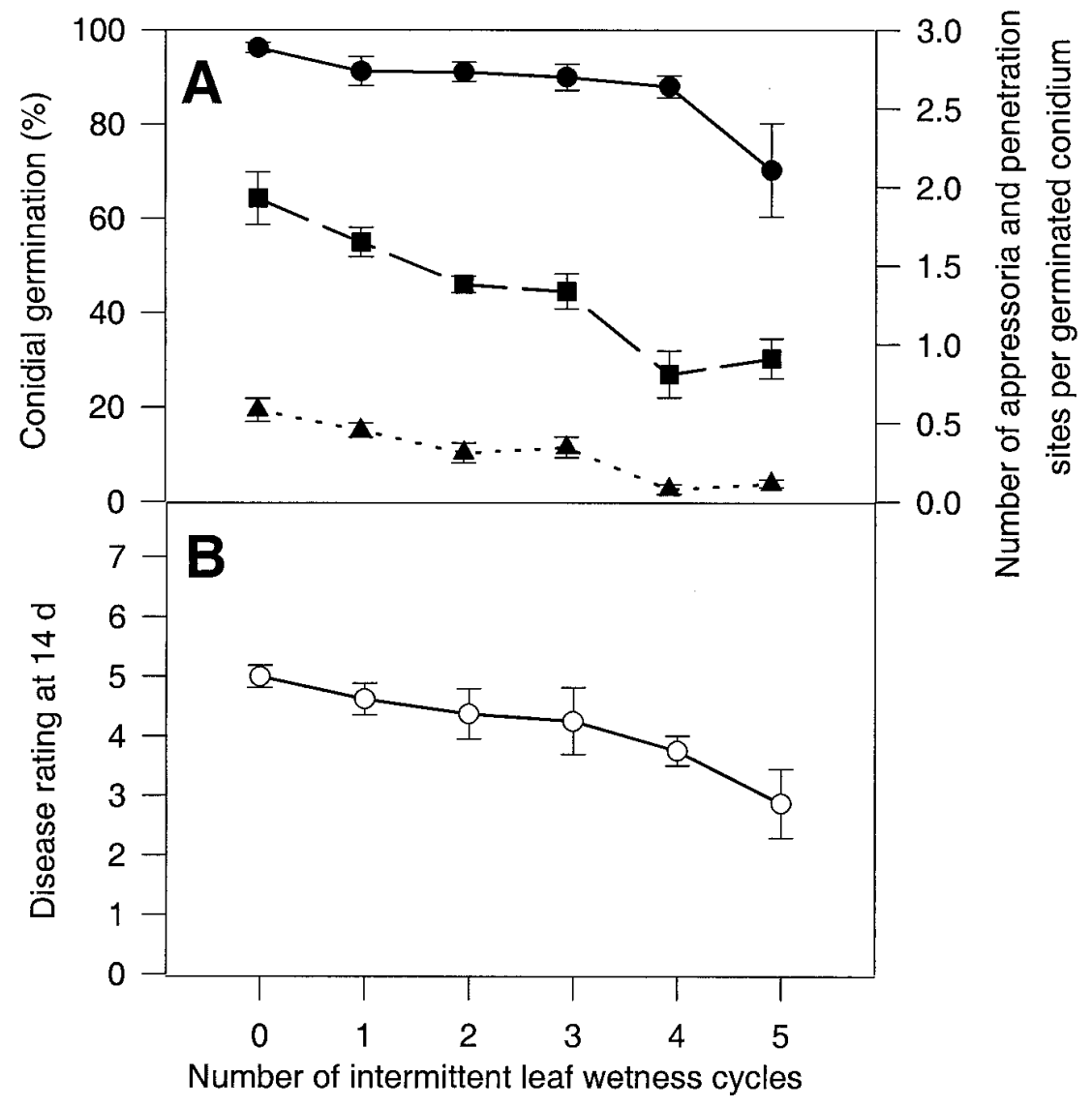

Fig. 4. Effect of increasing numbers of $30 \mathrm{~min}$ wet/30 min dry intermittent leaf wetness cycles before a 24-h continuous leaf wetness period on germination (U) of conidia of Alternaria cirsinoxia, appressoria formation ( $\mathbf{\square}$ ), and penetration of leaves ( $\mathbf{\Delta}$ ) of Canada thistle (A), and on disease ratings on whole plants 14 days after inoculation (B). Data presented are untransformed means and bars indicate standard error. ration for all stages of infection of Canada thistle, and for a minimum of $8 \mathrm{~h}$ to cause disease on this weed. A. cirsinoxia also exhibited a degree of tolerance to a range of temperatures, as well as to repeated cycles of desiccation and rehydration. This information can be used to assess the potential of this fungus as a bioherbicide for Canada thistle.

Rotem (12) stated that free water is essential for germination and infection by Alternaria spp. This study indicates that free water is optimal, but not essential, for infection of Canada thistle by A. cirsinoxia, as conidia germinated well at $98 \%$ $\mathrm{RH}$ and penetrated leaves at $100 \% \mathrm{RH}$, with the highest incidence of leaf penetration when free water was present on the leaves. Rotem (12) warned that reports of germination of Alternaria spp. below saturation should be viewed with some caution, since microscopic condensation of water may occur around conidia. This may be true for A. cirsinoxia at 98 and $100 \%$ RH, although it is impossible to determine the precise nature of moisture available to conidia on the surface of transpiring leaves. Cooke and Whipps (2) suggested that during establishment of infection by fungal pathogens, spore germination requires more specific environmental conditions than subsequent stages in the development of infection. However, pregerminated conidia of $A$. cirsinoxia still required at least $100 \% \mathrm{RH}$, and preferably free water, to form at least one appressorium per conidium, and to penetrate leaves. Although conidia of Alternaria spp. are generally wind-dispersed (12), A. cirsinoxia was inoculated onto Canada thistle in a water suspension. The possibility of conidial germination being initiated by the water in the inoculation procedure was minimized by applying the inoculum immediately after preparation, and allowing the leaves to dry before applying the humidity treatments.

In the presence of free water on the leaves, A. cirsinoxia germinated and infected Canada thistle at all temperatures tested between 10 and $30^{\circ} \mathrm{C}$. These results are typical for Alternaria spp., which do not have fastidious temperature requirements for infection (12). In Saskatchewan, the mean maximum and minimum temperatures from June to August are approximately 25 and $10^{\circ} \mathrm{C}$, respectively, although extreme temperatures of up to $40^{\circ} \mathrm{C}$ and below freezing may occur (4). Provided free moisture is available, temperatures within the average range for Saskatchewan during the growing season should not be a limiting factor to infection of Canada thistle by A. cirsinoxia.

Since the presence of free water on the leaf surface is optimal for infection of Canada thistle by A. cirsinoxia, further research is needed to determine the duration of free water necessary to establish infection on Canada thistle. The duration 
of leaf wetness required by Alternaria spp. is highly variable, ranging between 3 and $72 \mathrm{~h}$ (12). A. cirsinoxia can germinate within $2 \mathrm{~h}$ continuous leaf wetness and initiate leaf penetration within $4 \mathrm{~h}$ continuous leaf wetness, but a continuous leaf wetness period of $8 \mathrm{~h}$ was needed to establish visible symptoms of infection on Canada thistle. In this study, leaf wetness requirements of $A$. cirsinoxia were tested within the optimal temperature range for $A$. cirsinoxia. However, in the field, temperature fluctuations outside the optimal range may slow the rate of infection and increase the duration of leaf wetness required. This has been reported for other Alternaria spp. $(3,7,11)$. For example, a 3$\mathrm{h}$ minimum wet period was required by Alternaria brassicae (Berk.) Sacc to infect leaves of oilseed rape (Brassica campestris L. ssp. oleifera) at 20 to $25^{\circ} \mathrm{C}$, but at $5^{\circ} \mathrm{C}$ the minimum wet period increased to 12 to $24 \mathrm{~h}$ (11). Examining the leaf wetness/temperature interactions for $A$. cirsinoxia is another area requiring further evaluations. For example, evening inoculations are more likely to coincide with dew periods than midday inoculations, but may also coincide with considerably cooler temperatures.

Continuous leaf wetness periods of $8 \mathrm{~h}$ or more are infrequent in the Canadian prairies. If inoculation does not coincide with sufficient moisture for infection, it may be necessary for $A$. cirsinoxia to germinate and infect Canada thistle during intermittent leaf wetness periods, or to at least survive these periods until prolonged moisture does occur. Although exposure to an increasing number of 30 min intermittent leaf wetness cycles resulted in a reduction in infection, A. cirsinoxia was still able to incite disease on Canada thistle after five intermittent leaf wetness cycles. This study also indicated that germinated conidia are more sensitive to subsequent dry periods than ungerminated conidia, since exposure to $4 \mathrm{~h}$ intermittent leaf wetness periods was more limiting to establishment of infection than exposure to 1 and $2 \mathrm{~h}$ intermittent leaf wetness periods. Germ tubes are delicate and highly sensitive to suboptimal conditions (2). Desiccation may be lethal for both the germ tube,

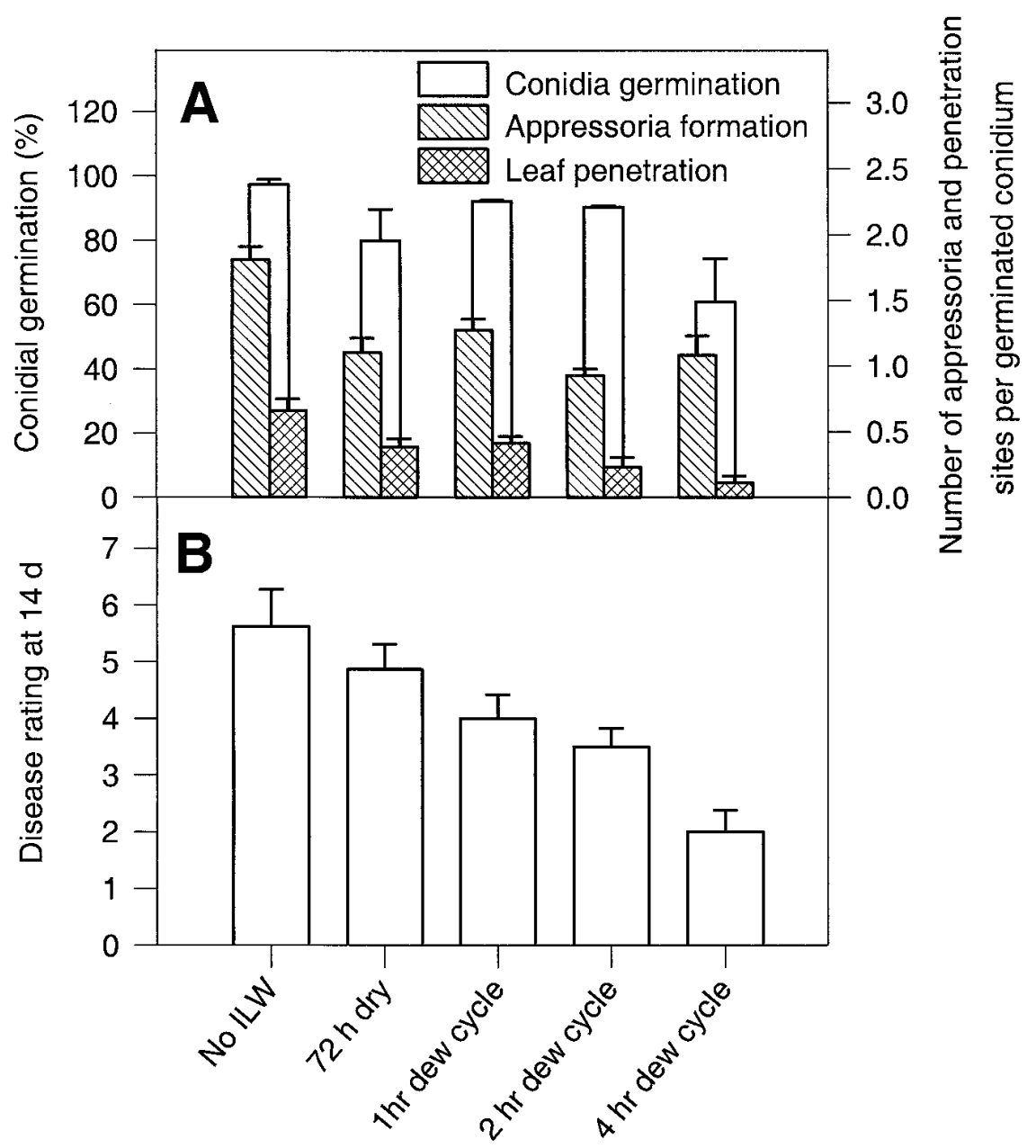

Fig. 5. Effect of no intermittent leaf wetness (ILW), a 72-h dry period, and three cycles of $1 \mathrm{~h}$ dew/23 $\mathrm{h}$ dry, $2 \mathrm{~h}$ dew/22 h dry, and $4 \mathrm{~h}$ dew/20 h dry before a $24 \mathrm{~h}$ continuous leaf wetness period on germination of conidia of Alternaria cirsinoxia, appressoria formation, and penetration of leaves of Canada thistle (A), and on disease ratings on whole plants 14 days after inoculation (B). Data presented are untransformed means and bars indicate standard error. and the conidial cell from which it arose. Tolerance of A. cirsinoxia to repeated wetting and drying may be greater if the intermittent leaf wetness periods are too brief to permit extensive germination. The ability of A. cirsinoxia to survive and infect its host after several, short intermittent leaf wetness cycles is typical for Alternaria spp. in general (12). However, in a field situation other factors such as exposure to prolonged, direct solar radiation and dry, warm winds, need to be considered when predicting the short-term survival of $A$. cirsinoxia on the phylloplane.

A. cirsinoxia has promising characteristics as a potential bioherbicide, such as survival during less than optimal moisture conditions, and nonspecific temperature requirements. However, extensive infection of Canada thistle in the Canadian prairie environment is unlikely unless prolonged dew or rainfall provide sufficient moisture to complete the infection process. In Saskatchewan, measurable precipitation typically falls on less than 1 in 5 days (5), which means inoculations should be timed to coincide with rainfall in order to achieve maximum rates of infection and prevent reduction in conidial viability. The ecological limitations of bioherbicides can be mitigated to some extent by formulation and application technology (6), but artificially reducing the dependency of $A$. cirsinoxia on the presence of dew presents a major challenge. Because of the relatively high moisture requirements for infection of A. cirsinoxia, compared to typical moisture conditions, A. cirsinoxia may have limited potential as a bioherbicide in the semi-arid regions of the Canadian prairies, except within irrigated cropping systems. This fungus may have greater potential as a bioherbicide for Canada thistle infestations in regions with higher rainfall and humidity levels, for example Ontario, or the maritime provinces of Canada. As an area for further study, other isolates of A. cirsinoxia could be collected and evaluated to determine whether strains with lower moisture requirements for infection of Canada thistle can be identified.

\section{ACKNOWLEDGMENTS}

We thank Anita Lemke for technical assistance and Jon Geissler for production of conidia. Financial support for this study was provided by MicroBio RhizoGen Corporation and an Agriculture and Agri-Food Canada Matching Investment Initiative.

\section{LITERATURE CITED}

1. Charudattan, R. 1991. The mycoherbicide approach with plant pathogens. Pages 24-57 in: Microbial Control of Weeds. D. O. TeBeest, ed. Chapman and Hall, New York.

2. Cooke, R. C., and Whipps, J. M. 1993. Ecophysiology of Fungi. Blackwell Scientific Publications, Oxford, UK.

3. Degenhardt, K. J., Petrie, G. A., and Morrall, R. A. A. 1982. Effects of temperature on spore germination and infection of rapeseed by Alternaria brassicae, A. brassicicola, and A. raphani. Can. J. Plant Pathol. 4:115-118. 
4. Environment Canada. 1990. Canadian climate normals (1961-1990), temperature and precipitation: prairie provinces. Canadian Climate Program Publication.

5. Fung, K., Barry, B., and Wilson, M. 1999. Atlas of Saskatchewan. University of Saskatchewan, Saskatoon.

6. Green, S., Stewart-Wade, S. M., Boland, G. J., Teshler, M. L., and Liu, S. H. 1998. Formulating micro-organisms for biological control of weeds. Pages 249-282 in: Plant-Microbe Interactions and Biological Control. G. J. Boland and L. D Kuykendall, eds. Marcell Dekker, New York.

7. Hong, C. X., and Fitt, B. D. L. 1995. Effects of inoculum concentration, leaf age and wetness period on the development of dark leaf and pod spot (Alternaria brassicae) on oilseed rape (Brassica napus). Ann. App. Biol. 127:283-295.

8. Horsfall, J. G., and Barratt, R. W. 1945. An improved grading system for measuring plant diseases. Phytopathology 35:655.

9. Keane, P. J., Limongiello, N., and Warren, M. A. 1988. A modified method for clearing and staining leaf-infecting fungi in whole leaves. Aust. Plant Pathol. 17:37-38.

10. Moore, R. J. 1975. The biology of Canadian weeds. 13. Cirsium arvense (L.) Scop. Can. J. Plant Sci. 55:1033-1048.

11. Mridha, M. A. U., and Wheeler, B. E. J. 1993. In vitro effects of temperature and wet periods on infection of oilseed rape by Alternaria alternata. Plant Pathol. 42:671-675.
12. Rotem, J. 1994. The Genus Alternaria. Biology, Epidemiology, and Pathogenicity. APS Press, St. Paul, MN.

13. Simmons, E. G., and Mortensen, K. 1997. Alternaria cirsinoxia Simmons \& Mortensen, sp. nov. No. 218 in: Alternaria, Themes and Variations. Mycotaxon 53:72-76.

14. Thomas, A. G., Wise, R. F., Frick, B. L., and Juras, L. T. 1996. Saskatchewan Weed Survey. Cereal, oilseed and pulse crops. Agriculture and Agri-Food Canada Weed Survey Series Publication 96-1, Saskatoon, SK.

15. Walker, L. 1980. Production of spores for field studies. Adv. Agric. Tech. 12:1-5.

16. Winston, P. W., and Bates, D. H. 1960. Saturated salt solutions for the control of humidity in biological research. Ecology 41:232-237. 